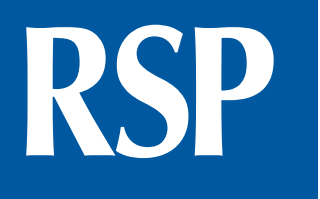

http://www.rsp.fsp.usp.br/
Revista de Saúde Pública

\title{
Características das mulheres que não consultam com médico: estudo de base populacional
}

Juvenal Soares Dias-da-Costa ${ }^{1,11}$, Annie Pozeczek Koltermann ${ }^{1, \prime \prime \prime}$, Bruna Cappellesso', Josiele Flores Lisowski', Maiton Bernardelli', Paula Brustolin Xavier',IV, Talita Donatti', Tissiani Morimoto ${ }^{\prime \prime}$, , Fernanda Souza de Bairros', Maria Teresa Anselmo Olinto'

' Universidade do Vale do Rio dos Sinos. Programa de Pós-Graduação em Saúde Coletiva. São Leopoldo, RS, Brasil " Universidade Federal de Pelotas. Faculdade de Medicina. Pelotas, RS, Brasil

III Universidade Feevale. Novo Hamburgo, RS, Brasil

Iv Universidade do Oeste de Santa Catarina. Área das Ciências da Vida. Joaçaba, SC, Brasil

$\checkmark$ Centro Universitário Ritter dos Reis. Curso de Fisioterapia. Porto Alegre, RS, Brasil

\section{RESUMO}

OBJETIVO: Analisar a prevalência de não consultar com médico no período de um ano.

MÉTODOS: Estudo transversal de base populacional, incluindo mulheres de 20 a 60 anos, residentes na zona urbana de São Leopoldo, RS, em 2015. A associação entre as variáveis e o desfecho foi avaliada por meio das razões de prevalência e dos intervalos de $95 \%$ de confiança (IC95\%). A análise ajustada foi realizada por meio da regressão de Poisson com variância robusta.

RESULTADOS: Entre as 1.127 mulheres participantes do estudo, 954 (84,6\%; IC95\% 82,5-86,7) referiram consultar com médico no ano anterior à entrevista, 173 (15,4\%; IC95\% 13,2-17,5) não consultaram. As mulheres inseridas nas classes econômicas D e E, com menor idade, e fumantes apresentaram maiores prevalências de não consulta médica. As participantes com hipertensão arterial tiveram maior prevalência de consultas.

CONCLUSÕES: Não houve a esperada evolução no sistema local de saúde, apesar do surgimento das políticas implantadas nesse período. É necessário provimento da atenção para os indivíduos em condições socioeconômicas menos favorecidas e para as mulheres mais jovens.

DESCRITORES: Mulheres. Aceitação pelo Paciente de Cuidados de Saúde. Serviços de Saúde,

utilização. Atenção Primária à Saúde. Acesso aos Serviços de Saúde. Assistência Ambulatorial.

Recebido: 15 abr 2017

Aprovado: 16 out 2017

Como citar: Dias-da-Costa JS, Koltermann AP, Cappellesso B, Lisowski JF, Bernardelli M, Xavier $\mathrm{PB}$, et al. Características das mulheres que não consultam com médico: estudo de base. Rev Saude Publica. 2018;52:54.

Copyright: Este é um artigo de acesso aberto distribuído sob os termos da Licença de Atribuição Creative Commons, que permite uso irrestrito, distribuição e reprodução em qualquer meio, desde que o autor e a fonte

originais sejam creditados.

Juvenal Soares Dias da Costa

Avenida Unisinos, 950

93022-000 São Leopoldo, RS, Brasil

E-mail: episoares@terra.com.br 


\section{INTRODUÇÃO}

O acesso universal e gratuito aos serviços e ações de saúde no modelo de saúde brasileiro foi garantido pela Lei Orgânica da Saúde ${ }^{a}$. A implantação do Sistema Único de Saúde (SUS) ocorreu em um movimento de descentralização, proporcionando maior autonomia para os municípios, e envolvendo a participação social ${ }^{1}$. Para a ampliação da atenção básica, que tinha como objetivo o acesso universal e referência para níveis mais complexos de cuidados, o Ministério da Saúde formulou uma série de programas. Entre eles, está a Estratégia de Saúde da Família (ESF), que proporcionou maior cobertura da população ${ }^{1,2}$.

Estudos que avaliaram o acesso aos serviços de saúde evidenciaram avanços após o movimento de Reforma Sanitária e implantação do SUS ${ }^{3-5}$. Entre 1981 e 2008, o número de indivíduos que utilizaram a atenção básica aumentou aproximadamente $450 \%^{1}$. Ao se comparar os dados da Pesquisa Nacional por Amostra de Domicílios (PNAD) entre 1998 e 2008, foi constatado que a probabilidade de ter pelo menos uma visita médica nos últimos 12 meses tornou-se mais equitativa ao longo do tempo, e os fatores que se associavam à maior equidade incluíam as necessidades de saúde, escolaridade e vínculo com a ESF${ }^{6}$. Atualmente, o Brasil possui uma proporção de cobertura de ESF de 64,3\%, com estimativa de cobertura populacional de 124.773 .082 indivíduos e 266.583 agentes comunitários de saúde atuando no sistema de saúde ${ }^{\mathrm{b}}$. Especificamente em relação ao uso de serviços de saúde, estudo que utilizou a PNAD de 1998, mostrou que as mulheres consultavam mais do que os homens. Observou-se ainda que mulheres acima de 50 anos, aposentadas ou donas de casa, e com maior renda, consultavam mais ${ }^{3}$. Outro estudo com dados das PNAD de 2003 e 2008 constatou que as mulheres consultavam mais do que os homens. Mostrou também que as mulheres acima de 50 anos, com maior renda e com maior morbidade autorreferida, também se consultam mais 5 .

Foram desenvolvidos dois estudos transversais de base populacional, verificando o uso de serviços de saúde em São Leopoldo, RS. Em 2003, estudo que arrolou 1.022 mulheres de 20 a 60 anos apontou que aquelas inseridas nas classes econômicas C, D e E, com pior escolaridade e menor renda, apresentaram menor utilização dos serviços de saúde, evidenciando iniquidades entre a população mais vulnerável ${ }^{7}$. Por sua vez, outro estudo realizado em 2007, com 1.098 indivíduos de ambos os sexos com 20 a 69 anos, mostrou que os participantes nas categorias intermediárias de renda, escolaridade e classe econômica, procuravam menos os serviços de saúde, sugerindo avanços no sistema local de saúde em relação às populações mais necessitadas8.

Desta forma, entender a demanda e o uso de serviços de saúde com base em estudos epidemiológicos permite o conhecimento da realidade local, identificação de populações vulneráveis e instrumentalização da gestão para planejamento e provimento de cuidados adequados.

a Brasil. Lei no 8.080 , de 19 de setembro de 1990. Lei Orgânica da Saúde. Dispõe sobre as condições para a promoção, proteção e recuperação da saúde, a organização e o funcionamento dos serviços correspondentes e dá outras providências. Diario Oficial Uniao. 20 set. 1990; Seção 1:18055.

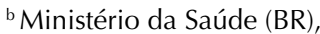
Secretaria de Atenção à Saúde, Departamento de Atenção Básica, Coordenação Geral de Gestão da Atenção Básica. Histórico de cobertura de Saúde da Família. Brasília (DF); 2016 [citado 10 dez 2016]. Disponível em: http://dab.saude.gov.br/dab/ historico_cobertura_sf/historico_ cobertura_sf_relatorio.php
Portanto, este estudo teve como objetivo analisar a prevalência de não consultar com médico no período de um ano em São Leopoldo, RS.

\section{MÉTODOS}

Estudo transversal de base populacional, com uma amostra representativa das mulheres entre 20 e 69 anos, residentes na área urbana de São Leopoldo, RS. Este artigo foi um recorte de um projeto de pesquisa intitulado "Condições de vida e saúde de mulheres adultas: estudo de base populacional no Vale do Rio dos Sinos - Avaliação após 10 anos”, realizado pela Universidade do Vale do Rio dos Sinos entre fevereiro e outubro de 2015. Esse estudo foi uma replicação de estudo semelhante conduzido em 2003, de forma que seus resultados pudessem ser comparados. O cálculo do tamanho de amostra foi realizado no programa EpiInfo 6.0. Foram estimados a partir da população feminina de São Leopoldo, com inúmeros desfechos, incluindo hábitos de vida, aspectos nutricionais, psicológicos, procedimentos preventivos, métodos contraceptivos, morbidades e uso de serviços de saúde. Optou-se pelo 
resultado que exigia maior tamanho de amostra. O desfecho que exigiu maior tamanho de amostra foi prevalência de exame citopatológico atrasado $(5,7 \%)^{9}$, com erro amostral de $3 \%$, acrescentando-se $25 \%$ para perdas e controle de fatores de confusão, totalizando 1.281 mulheres. O tamanho de amostra para não utilização de serviço de saúde ambulatoriais foi estimado a partir da prevalência do desfecho em não expostos $(6,8 \%)^{7}$, com razão de nãoexpostos para expostos de 1:2, razão de risco de 2,0, nível de confiança de $95 \%$ e poder de 80\% foi de 945 indivíduos.

A amostra foi sistemática. Os 371 setores censitários da região urbana de São Leopoldo foram classificados em ordem decrescente a partir do setor com maior "valor de rendimento nominal mensal das pessoas de 10 ou mais anos (com ou sem rendimento)", segundo o Instituto Brasileiro de Geografia e Estatística (IBGE) c. Foram selecionados 45 setores censitários. Em cada conglomerado, foi sorteado o quarteirão e a esquina para iniciar a pesquisa. A cada domicílio visitado, saltavam-se duas residências até incluírem 36 moradias em cada setor. Todas as mulheres que preenchiam os critérios de inclusão foram entrevistadas. Foram aplicados questionários padronizados, pré-codificados e pré-testados semelhantes à versão do estudo anteriormente realizado, com perguntas abertas e fechadas às participantes. Foram coletadas medidas de peso e altura das participantes por meio de balanças e antropômetros portáteis.

Mulheres que não residiam no domicílio sorteado, gestantes e aquelas com deficiência mental foram excluídas do estudo.

Os entrevistadores foram submetidos ao programa de treinamento para padronização da aplicação dos instrumentos. Foram realizadas mais duas tentativas em dias e horários diferentes no caso de domicílios fechados com moradores ausentes.

O controle de qualidade foi realizado em uma amostra aleatória de $10 \%$ das pessoas incluídas no estudo, com o intuito de avaliar a validade interna da pesquisa.

O desfecho foi não ter realizado nenhuma consulta médica no ano anterior à entrevista $\mathrm{e}$ foi avaliado pela pergunta "quantas vezes você se consultou com o médico desde o <mês> do ano passado?".

As variáveis independentes foram classificadas como demográficas, socioeconômicas, hábitos de vida e algumas morbidades.

As variáveis demográficas foram: idade (categorizada a cada dez anos), cor da pele autorreferida (branca e não branca), e situação conjugal (casada/em união ou vive sozinha; solteira, separada/divorciada; viúva).

Como variáveis socioeconômicas foram utilizadas: escolaridade ( $\geq 15$ anos; 11 a 14 anos; oito a 10 anos; cinco a sete anos; $\leq 4$ anos); renda familiar per capita em salários mínimos (> 3 salários mínimos; de um a três salários mínimos; < 1 salário mínimo); e classificação econômica (A; B; C; D+E) segundo o critério de classificação econômica proposta pela Associação Brasileira de Empresas de Pesquisa (ABEP)d.

Fundação Instituto Brasileiro de Geografia e Estatística. Censo Demográfico 2010. Rio de Janeiro: IBGE; 2010 [citado 12 dez 2016]. Disponível em: https://censo2010.ibge.gov.br/

${ }^{\mathrm{d}}$ Associação Brasileira de Empresas de Pesquisa. Critério de Classificação Econômica Brasil. São Paulo: ABEP; 2016 [citado 12 dez 2016]. Disponível em: http://www.abep.org/ codigosguias/ABEP_CCEB.pdf

e The IPAQ Group. International Physical Activity Questionnaire - IPAQ [citado $10 \mathrm{dez} 2016]$. Disponível em: https://sites. google.com/site/theipaq/

As variáveis que representaram os hábitos de vida foram: tabagismo (não fumante; ex-fumantes; fumantes); consumo excessivo de álcool (não; sim); e atividade física (sim; não). O consumo de álcool foi estabelecido a partir da frequência, do tipo de bebida e da quantidade ingerida, classificado como excessivo quando igual ou maior de $30 \mathrm{~g}$ de etanol/dia. As participantes foram consideradas fisicamente ativas quando atingiam no mínimo 150 minutos de atividades físicas verificadas por meio do IPAQ ${ }^{\mathrm{e}}$ - versão curta.

Foram incluídas na análise algumas morbidades como excesso de peso (não; sim); diabetes diagnosticada por médico referido pelo paciente (não; sim); hipertensão arterial sistêmica referida (não; sim); e transtornos psiquiátricos menores (não; sim). O estado nutricional dos pacientes foi verificado mediante o índice de massa corporal (IMC). Foi considerado excesso de peso quando o IMC atingiu valor igual ou maior do que $25 \mathrm{~kg} / \mathrm{m}^{2}$. A presença de transtornos psiquiátricos menores foi verificada mediante a aplicação do SelfReporting Questionaire(SRQ-20). 
Esse instrumento estava constituído por 20 perguntas dicotômicas. As participantes com escore com valor de sete ou mais foram consideradas com transtornos psiquiátricos menores.

A entrada de dados foi realizada em duplicata para se evitar erros de digitação. Foi feita a caracterização da amostra.

A análise bruta apontou as razões de prevalência, com respectivos intervalos de 95\% de confiança e testes estatísticos. A análise ajustada foi realizada mediante regressão de Poisson com variância robusta, seguindo modelo hierarquizado. As variáveis que obtiveram $p=0,20$ na análise bruta foram selecionadas para a análise ajustada, permanecendo no modelo aquelas com significância estatística de $<<0,05$. O modelo hierarquizado de análise foi composto pelas variáveis demográficas (idade, cor da pele, situação conjugal) e socioeconômicas (escolaridade, classe econômica, renda per capita em salários mínimos) no primeiro nível, as quais determinavam as variáveis que representavam os hábitos de vida (tabagismo, atividade física e consumo excessivo de álcool). No terceiro nível, determinadas pelas variáveis dispostas nos níveis superiores, situaram-se algumas morbidades (excesso de peso, diabetes mellitus, hipertensão arterial e transtornos psiquiátricos menores). Todas as variáveis determinavam o desfecho. Não se incluiu a variável escolaridade na análise ajustada pela possível colinearidade, uma vez que estava contida na classe econômica e 51,8\% das entrevistadas afirmaram ser a chefe da família.

A análise estatística foi obtida nos programas SPSS e Stata 12. O efeito do delineamento foi estimado no programa Stata, com valor de 0,95 e não foi considerado na análise. O Projeto foi submetido e aprovado pelo Comitê de Ética e Pesquisa da Universidade do Vale do Rio dos Sinos (Protocolo 653.394, em 20/5/2014). Todos os participantes assinaram termo de consentimento livre e esclarecido.

\section{RESULTADOS}

Entre as 1.127 mulheres participantes do estudo, 84,6\% (IC95\% 82,5-86,7) referiram consultar com médico no ano anterior à entrevista, 15,4 (IC95\% 13,2-17,5) não consultaram. O percentual de perdas e recusas atingiu $11,9 \%$.

Houve distribuição homogênea entre as categorias de idade, com predomínio da faixa etária de 40 a 49 anos (24,5\%). A amostra foi composta em sua maioria por mulheres brancas (74,4\%), casadas ou em união estável (63,9\%), com oito anos ou mais de estudo (59,4\%), pertencentes à classe econômica C (53,1\%), e com renda familiar per capita inferior a um salário mínimo (60,8\%) (Tabela 1).

As maiores prevalências de não consulta médica ocorreram nas mulheres mais jovens na análise bruta. As mulheres com menos de 14 anos de estudo apresentaram maior prevalência de não consultar, porém esse efeito não foi observado na categoria de até quatro anos. As classes econômicas D e E apresentaram maiores prevalências de não consultar quando comparadas às mulheres inseridas na classe $\mathrm{A}$. As mulheres com renda familiar per capita menor do que um salário mínimo apresentaram maior prevalência de não consultas. Porém, os intervalos de confiança incluíram o valor unitário, apesar do resultado do teste ser significativo, encaminhando a variável ao modelo ajustado. Não foram evidenciadas diferenças estatisticamente significativas nas variáveis: cor da pele e estado civil (Tabela 1).

A maioria das mulheres não fumava (59,0\%), não consumia álcool em excesso $(97,0 \%)$ e não praticava atividade física $(85,6 \%)$. Apresentaram excesso de peso $66,1 \%$ das participantes, $28,1 \%$ hipertensão arterial, 8,1\% diabetes mellitus e 39,8\% transtornos psiquiátricos menores (Tabela 2).

As mulheres fumantes apresentaram maior prevalência do desfecho na análise bruta. As mulheres classificadas com consumo de álcool excessivo aparentemente apresentavam maior prevalência de não consulta, mas sem diferença no teste estatístico. Por sua vez, as mulheres com excesso de peso, hipertensão arterial e diabetes mellitus estavam protegidas de não consultarem. Os transtornos psiquiátricos menores não estavam associados ao desfecho (Tabela 2). 
Tabela 1. Características demográficas e socioeconômicas e análise bruta da prevalência de não consulta médica entre mulheres. São Leopoldo, RS, Brasil, 2015.

\begin{tabular}{|c|c|c|c|c|c|c|}
\hline Variável & $\mathbf{n}$ & $\%$ & $\begin{array}{c}\text { Prevalência de } \\
\text { não uso }(\%)\end{array}$ & $\mathbf{R P}$ & IC95\% & $\mathbf{p}$ \\
\hline Idade (anos) & & & & & & $<0,001$ \\
\hline $20-29$ & 216 & 19,2 & $51(29,3)$ & 1 & & \\
\hline $30-39$ & 244 & 21,7 & $44(25,3)$ & 0,76 & 0,53-1,09 & \\
\hline $40-49$ & 276 & 24,5 & $41(23,6)$ & 0,63 & $0,43-0,91$ & \\
\hline $50-59$ & 228 & 20,2 & $23(13,2)$ & 0,43 & $0,27-0,67$ & \\
\hline $60-69$ & 163 & 14,5 & $15(8,6)$ & 0,39 & $0,23-0,67$ & \\
\hline Cor & & & & & & 0,78 \\
\hline Não branca & 288 & 25,6 & $46(16,0)$ & 1 & & \\
\hline Branca & 839 & 74,4 & $128(15,3)$ & 0,95 & $0,69-1,29$ & \\
\hline Situação conjugal & & & & & & 0,44 \\
\hline Solteira & 227 & 20,1 & $43(18,9)$ & 1 & & \\
\hline Casada/Em união & 720 & 63,9 & $105(14,6)$ & 0,79 & $0,57-1,09$ & \\
\hline Separada/Divorciada & 110 & 9,8 & $16(14,5)$ & 0,78 & $0,46-1,33$ & \\
\hline Viúva & 70 & 6,2 & $10(14,3)$ & 0,76 & $0,40-1,43$ & \\
\hline Escolaridade (anos) & & & & & & $<0,01$ \\
\hline$\geq 15$ & 110 & 9,8 & $6(5,5)$ & 1 & & \\
\hline $11-14$ & 360 & 32,0 & $62(17,2)$ & 3,16 & $1,40-7,10$ & \\
\hline $8-10$ & 198 & 17,6 & $40(20,2)$ & 3,61 & $1,58-8,26$ & \\
\hline $5-7$ & 253 & 22,5 & $42(16,6)$ & 3,04 & $1,33-6,95$ & \\
\hline $0-4$ & 204 & 18,1 & $24(11,8)$ & 2,16 & $0,91-5,12$ & \\
\hline \multicolumn{7}{|l|}{ Classe econômica } \\
\hline A & 44 & 3,9 & $2(4,5)$ & 1 & & $<0,001$ \\
\hline B & 346 & 30,9 & $37(10,7)$ & 2,35 & $0,59-9,43$ & \\
\hline $\mathrm{C}$ & 595 & 53,1 & $101(17,1)$ & 3,73 & $0,95-14,64$ & \\
\hline $\mathrm{D}+\mathrm{E}$ & 136 & 12,1 & $33(24,3)$ & 5,34 & $1,34-21,36$ & \\
\hline \multicolumn{5}{|c|}{ Renda per capita em salários mínimos (SM) } & & $<0,001$ \\
\hline 3 ou mais & 66 & 6,1 & $7(10,6)$ & 1 & & \\
\hline 1 a 2,99 & 361 & 33,1 & $32(8,9)$ & 0,83 & $0,38-1,81$ & \\
\hline Menos de 1 & 663 & 60,8 & $127(19,3)$ & 1,81 & $0,88-3,71$ & \\
\hline
\end{tabular}

RP: razão de prevalência

Tabela 2. Características de hábitos de vida e morbidades e análise bruta da prevalência de não consulta médica entre mulheres. São Leopoldo, RS, Brasil, 2015.

\begin{tabular}{|c|c|c|c|c|c|c|}
\hline Variável & $\mathrm{n}$ & $\%$ & $\begin{array}{l}\text { Prevalência de } \\
\text { não uso (\%) }\end{array}$ & $\mathbf{R P}$ & IC95\% & p \\
\hline Tabagismo & & & & & & 0,001 \\
\hline Não fuma & 661 & 59,0 & $90(13,6)$ & 1 & & \\
\hline Ex-fumante & 253 & 22,6 & $32(12,6)$ & 0,93 & $0,64-1,35$ & \\
\hline Fumante & 207 & 18,5 & $207(24,6)$ & 1,81 & $1,33-2,46$ & \\
\hline Consumo excessivo de álcool & & & & & & 0,17 \\
\hline Não & 1.086 & 97,0 & $166(15,3)$ & 1 & & \\
\hline Sim & 34 & 3,0 & $8(23,5)$ & 1,54 & $0,83-2,87$ & \\
\hline Atividade física & & & & & & 0,25 \\
\hline Sim & 162 & 14,4 & $20(12,3)$ & 1 & & \\
\hline Não & 965 & 85,6 & $154(16,0)$ & 1,29 & $0,84-2,00$ & \\
\hline Excesso de peso & & & & & & 0,001 \\
\hline Não & 380 & 33,9 & $78(20,5)$ & 1 & & \\
\hline Sim & 741 & 66,1 & $95(12,8)$ & 0,62 & $0,47-0,82$ & \\
\hline Hipertensão arterial & & & & & & $<0,001$ \\
\hline Não & 810 & 71,9 & $153(18,9)$ & 1 & & \\
\hline Sim & 316 & 28,1 & $21(6,6)$ & 0,35 & $0,16-0,22$ & \\
\hline Diabetes mellitus & & & & & & $<0,01$ \\
\hline Não & 1.029 & 91,9 & $167(16,2)$ & 1 & & \\
\hline Sim & 91 & 8,1 & $5(5,5)$ & 0,34 & $0,14-0,19$ & \\
\hline Transtornos psiquiátricos & & & & & & 0,65 \\
\hline Não & 678 & 60,2 & $102(15,0)$ & 1 & & \\
\hline Sim & 449 & 39,8 & $72(16,0)$ & 1,06 & $0,81-1,40$ & \\
\hline
\end{tabular}

RP: razão de prevalência 
Tabela 3. Análise ajustada da prevalência de não consulta médica entre mulheres. São Leopoldo, RS, Brasil, 2015.

\begin{tabular}{|c|c|c|c|}
\hline Variável & RP ajustada & IC95\% & $\mathbf{p}$ \\
\hline Faixa etária $\left(\right.$ anos) $^{\mathrm{a}}$ & & & $<0,001$ \\
\hline $20-29$ & 1 & & \\
\hline $30-39$ & 0,76 & $0,53-1,09$ & \\
\hline $40-49$ & 0,68 & $0,47-0,98$ & \\
\hline $50-59$ & 0,42 & $0,26-0,68$ & \\
\hline $60-69$ & 0,42 & $0,25-0,72$ & \\
\hline Classe econômica $^{a}$ & & & $<0,05$ \\
\hline A & 1 & & \\
\hline $\mathrm{B}$ & 4,2 & $0,61-28,5$ & \\
\hline $\mathrm{C}$ & 5,76 & $0,84-39,5$ & \\
\hline $\mathrm{D}+\mathrm{E}$ & 7,20 & $1,03-50,3$ & \\
\hline Renda per capita em salários mínimos $(\mathrm{SM})^{\mathrm{a}}$ & & & 0,02 \\
\hline 3 ou mais & 1 & & \\
\hline 1 a 2,99 & 0,59 & $0,28-1,25$ & \\
\hline Menos de 1 & 1,00 & $0,47-2,11$ & \\
\hline Tabagismo $^{b}$ & & & 0,02 \\
\hline Não fuma & 1 & & \\
\hline Ex-fumante & 0,95 & $0,64-1,41$ & \\
\hline Fumante & 1,53 & $1,10-2,13$ & \\
\hline Consumo excessivo de álcool ${ }^{\mathrm{b}}$ & & & 0,79 \\
\hline Não & 1 & & \\
\hline Sim & 1,10 & $0,55-2,20$ & \\
\hline Excesso de peso ${ }^{c}$ & & & 0,06 \\
\hline Não & 1 & & \\
\hline Sim & 0,76 & $0,57-1,01$ & \\
\hline Hipertensão arterialc & & & $<0,001$ \\
\hline Não & 1 & & \\
\hline Sim & 0,40 & $0,24-0,66$ & \\
\hline Diabetes mellitus ${ }^{\mathrm{c}}$ & & & 0,25 \\
\hline Não & 1 & & \\
\hline Sim & 0,63 & $0,25-1,57$ & \\
\hline
\end{tabular}

RP ajustada: razão de prevalência ajustada

a Variáveis ajustadas entre si.

b Ajustadas para faixa etária, classe econômica, renda per capita em salários mínimos e entre si.

c Ajustadas para faixa etária, classe econômica, renda per capita em salários mínimos, tabagismo e entre si.

$\mathrm{Na}$ análise ajustada do primeiro nível, faixa etária, classe econômica e renda per capita mantiveram-se associadas ao desfecho não consulta médica. A partir dos 50 anos, constatou-se $58,0 \%$ de proteção para não consultas na comparação com as mulheres de 20 a 29 anos. A prevalência de não consultar entre as mulheres inseridas nas classes $\mathrm{D}$ e $\mathrm{E}$ foi 7,2 vezes maior em relação àquelas da classe econômica $A$. Apesar da imprecisão dos intervalos de confiança das categorias de renda, a variável foi mantida no modelo devido ao valor do teste. Mesmo ajustado para as variáveis do primeiro nível, as mulheres fumantes apresentaram maior prevalência do desfecho. Após ajuste para faixa etária, classe econômica, renda familiar, tabagismo e entre as variáveis do mesmo nível, as mulheres com hipertensão arterial continuavam protegidas de não consultar (Tabela 3).

\section{DISCUSSÃO}

Diversas políticas procuram melhorar as condições socioeconômicas da população no Brasil nos últimos $\operatorname{anos}^{10}$. O índice Gini, que reflete o nível de desigualdade social, diminuiu de forma importante e a implantação do SUS é apontada como catalisadora da melhoria nas condições de saúde da população brasileira ${ }^{11}$. Do ponto de vista de políticas de saúde, destacou-se a tentativa de fortalecer a atenção primária por meio da ESF. Certamente, foi uma forma de se ampliar o acesso e de se garantir a universalidade do atendimento, mostrando impressionante expansão e mudanças em indicadores de saúde ${ }^{12}$. Assim, esperava-se encontrar resultados positivos ao se replicar estudo transversal de base populacional sobre o uso de serviços de saúde em São Leopoldo no período de 10 anos. 
Foi possível comparar os resultados das variáveis socioeconômicas da presente investigação com outro estudo realizado em $2003^{7}$, ressaltando-se que a investigação anterior foi restrita à população de $20 \mathrm{a} 60$ anos.

Foram verificadas alterações em relação à comparação das variáveis socioeconômicas dos dois estudos. Em 2003, 66,9\% das participantes estavam distribuídas nas categorias de classe econômica C (39,3\%) e D+E (27,6\%). No presente estudo, 53,1\% das mulheres estavam inseridas na classe $\mathrm{C}$ e $12,1 \%$ na categoria $\mathrm{D}+\mathrm{E}$, o que corresponde a um aumento de $35,0 \%$ e uma diminuição de 56,1\%, respectivamente. Em 2003, as mulheres inseridas na categoria $\mathrm{D}+\mathrm{E}$ apresentavam maior prevalência de não consulta (16,7\%). No presente estudo, a prevalência de não consulta atingiu $24,3 \%$, indicando um aumento de $45,5 \%$. Isso representou um aumento das pessoas inseridas na classe $\mathrm{C}$, mas perdurou o efeito de não consultar nas mulheres de pior categoria de classe econômica.

Em 2003, 78,9\% das participantes relataram recebimento de até três salários mínimos, enquanto no presente estudo, esse contingente atingiu 93,9\%. Isso corresponde a um aumento de 19,0\%. Além disso, a categoria até um salário mínimo que abrangia 36,7\% das mulheres no primeiro estudo alcançou o percentual de $60,8 \%$ na presente investigação, representando um aumento de 65,6\%. Em 2003, as mulheres na pior categoria de renda apresentaram maior prevalência de não consulta. Contudo, os intervalos de confiança não confirmaram a diferença em relação ao desfecho no presente estudo.

Em 2003, 20,3\% das mulheres referiram até quatro anos de estudo. No presente estudo, esse percentual diminuiu para $18,1 \%$, com queda de $10,0 \%$.

As mudanças na distribuição da variável classe econômica e escolaridade com diminuição dos percentuais nas piores categorias podem ser entendidas como consequência de políticas sociais implantadas no país no período. Assim, o crescimento de participantes na classe C pode ser explicado pela posse de bens de consumo e de escolaridade. Contudo, mesmo após 10 anos, o sistema de saúde não superou as iniquidades constatadas em 2003.

No presente estudo, 15,4\% (IC95\% 13,2-17,5) das mulheres não consultaram com médico nos últimos 12 meses. No estudo com o mesmo desenho, mesma condução, realizado em 2003 com população semelhante, a prevalência de não utilização dos serviços ambulatoriais de saúde nos últimos 12 meses foi de 13,3\% (IC95\% 11,2-15,4)7. Contudo, o estudo anterior considerou qualquer utilização de serviço ambulatorial de saúde, diferentemente da investigação atual, em que se indagou especificamente sobre consulta médica. Mesmo assim, não houve a esperada evolução no sistema local de saúde, apesar do surgimento das políticas implantadas ao longo desse período (2003 a 2015).

Foram realizados outros estudos na região Sul do Brasil com resultados semelhantes aos do presente estudo. Em Santa Catarina, verificou-se prevalência de 76,0\% (IC95\% 73,6-78,4) de consulta médica na população adulta. Sendo assim, inferiu-se que a prevalência de não consulta médica na população foi de $24,0 \%$ e de $18,3 \%$ entre as mulheres ${ }^{13}$. No estudo de Bastos et al., conduzido em comunidade de baixa renda em Porto Alegre, RS, observou-se prevalência de consulta médica de 76,2\% (IC95\% 74,8-77,6) no último ano e 64,8\% (IC95\% 63,0-66,7) nos últimos três meses, maior entre as mulheres $(67,8 \%)$ do que entre os homens $(60,2 \%)^{14}$.

Estudos que analisaram as grandes pesquisas nacionais de saúde realizadas no país também apresentaram resultados semelhantes. Estudo de Macinko e Lima-Costa, avaliando as tendências de equidade horizontal na utilização dos serviços de saúde nos anos de 1998, 2003 e 2008 com dados da PNAD, mostrou que a probabilidade de ter pelo menos uma visita médica nos últimos 12 meses tornou-se mais equitativa ao longo do tempo ${ }^{6}$. Entretanto, a prevalência de consulta médica foi de 54,5\% em 1998,63,8\% em 2003 e 67,7\% em 2008, todas abaixo do percentual atingido no presente estudo 6 . Um dos achados do estudo foi que a maior desigualdade no uso do serviço está relacionada à renda, indicando iniquidade do sistema de saúde. Dados mais recentes, utilizando a Pesquisa Nacional de Saúde de 2013, apontaram que a prevalência de não visitar médico durante o último ano foi de $25,8 \%$ entre os entrevistados e $18,0 \%$ entre as mulheres ${ }^{15}$. 
No presente estudo, a prevalência de não consultar entre as mulheres pertencentes às classes $\mathrm{D}$ e E foi sete vezes maior em relação àquelas da classe A. No estudo transversal anteriormente realizado em São Leopoldo, identificou-se que mulheres inseridas na classe econômica D e E obtiveram 2,4 vezes menos utilização de serviços de saúde quando comparadas às da classe $\mathrm{A}^{7}$. Os resultados de ambos os estudos sugeriram que as mulheres mais vulneráveis possuíam maior dificuldade em acessar os serviços de saúde e que essa associação foi ainda mais evidente quando se buscava pelo atendimento médico.

Esses achados são condizentes com as comparações nacionais;todos evidenciando a persistência de iniquidades em saúde. Em estudo transversal realizado em Porto Alegre, as mulheres pertencentes às classes econômicas $\mathrm{C}, \mathrm{D}$ e E consultaram menos ${ }^{14}$. O desfecho de subutilização aplicado por Boccolini e Souza Júnior ${ }^{15}$ foi a ocorrência de resposta positiva em pelo menos uma combinação das seguintes perguntas: nunca consultou com médico; nunca consultou com dentista; e nunca mediu a pressão arterial ou glicose sanguínea ${ }^{15}$. Os resultados da análise ajustada mostraram maior subutilização nas classes sociais C, D e E, e nos indivíduos com menor escolaridade ${ }^{15}$. O presente estudo confirmou a persistência de indesejável iniquidade no sistema de saúde de São Leopoldo.

As mulheres das faixas etárias acima de 20 a 29 anos apresentaram menor probabilidade de não consulta médica, o que confirma os achados de outros estudos ${ }^{8,14,16}$. As doenças crônicas não transmissíveis são mais prevalentes nos indivíduos idosos ${ }^{16,17}$, o que exige maior intensidade de cuidados médicos. Além disso, os programas de prevenção secundários voltados à saúde da mulher contemplam prioritariamente faixas etárias mais avançadas.

Entre os achados do presente estudo verificou-se que as participantes fumantes apresentaram maior prevalência de não consultas médicas. Em estudo realizado em Lages, SC, Boing et al. ${ }^{13}$ também mostraram que os fumantes consultaram menos.

Estudos apontam que a presença de doenças crônicas não transmissíveis ou que a presença de mais morbidades implica aumento dos cuidados de saúde e consequentemente em mais consultas ${ }^{3,4}$. Entre as morbidades analisadas no presente estudo, constataram-se diferenças apenas entre as mulheres que apresentavam hipertensão arterial. No estudo realizado em 2003, as mesmas morbidades não foram associadas ao desfecho ${ }^{7}$. Contudo, outros estudos mostram maior utilização de serviços de saúde em pacientes com diabetes mellitus $^{13,18}$, transtornos psiquiátricos menores ${ }^{4,18}$ e hipertensão arterial ${ }^{19}$. Seria esperado que as participantes com essas morbidades referissem maior número de consultas. Assim, as descobertas do presente estudo apontam para a necessidade de implantação de ações programáticas para captar usuários com essas enfermidades.

Esses achados podem estar relacionados com a baixa cobertura em saúde. A proporção de cobertura populacional pela ESF em São Leopoldo era de 20,7\% em outubro de 2016. Conforme critérios de classificação de cobertura populacional pela ESF, valores inferiores a 30,0\% de cobertura podem ser classificados como incipientes ${ }^{19}$.

O presente estudo foi conduzido com o rigor necessário para as investigações de base populacional. Identificou mudanças na estrutura socioeconômica do município, constatando iniquidades no uso de consultas médicas. Destacou as características das mulheres que não consultavam com médicos, chamando a atenção para a necessidade de se apontar os cuidados direcionados para as doenças crônicas não transmissíveis. Assim, o presente estudo ressalta a conveniência de provimento da atenção para os indivíduos com condição socioeconômica menos favorecida e nas mulheres mais jovens.

\section{REFERÊNCIAS}

1. Paim J, Travassos C, Almeida C, Bahia L, Macinko J. The Brazilian health system: history, advances, and challenges. Lancet. 2011;377(9779):1778-97. https://doi.org/10.1016/S0140-6736(11)60054-8 
2. Szwarcwald CL, Damacena GN, Souza Júnior PRB, Almeida WS, Malta DC. Percepção da população brasileira sobre a assistência prestada pelo médico. Brasil, 2013. Cienc Saude Coletiva. 2016;21(2):339-50. https://doi.org/10.1590/1413-81232015212.19332015

3. Travassos C, Viacava F, Fernandes C, Almeida CM. Desigualdades geográficas e sociais na utilização de serviços de saúde no Brasil. Cienc Saude Coletiva. 2000;5(1):133-49. https://doi.org/10.1590/S1413-81232000000100012

4. Mendoza-Sassi R, Béria JU, Barros ADJ. Outpatient health service utilization and associated factors: a population-based study. Rev Saude Publica. 2003;37(3):372-8. https://doi.org/10.1590/S0034-89102003000300017

5. Silva PSC, Boing AF, Peres KG. Redução das desigualdades no uso de consultas médicas no Brasil: análise das regiões Nordeste e Sudeste entre 2003 e 2008. Rev Bras Epidemiol. 2015;18(1):248-61. https://doi.org/10.1590/1980-5497201500010019

6. Macinko J, Lima-Costa MF. Horizontal equity in health care utilization in Brazil, 1998-2008. Int J Equity Health. 2012;11:33. https://doi.org/10.1186/1475-9276-11-33

7. Dias da Costa JS, Presser AD, Zanotta AF, Ferreira DG, Perozzo G, Freitas IBA, et al. Utilização dos serviços ambulatoriais de saúde por mulheres: estudo de base populacional no Sul do Brasil. Cad Saude Publica. 2008;24(12):2843-51. https://doi.org/10.1590/S0102-311X2008001200013

8. Dias da Costa JS, Olinto MTA, Soares SA, Nunes MF, Bagatini T, Marques MC, et al. Utilização de serviços de saúde pela população adulta de São Leopoldo, Rio Grande do Sul, Brasil: resultados de um estudo transversal. Cad Saude Publica. 2011;27(5):868-76. https://doi.org/10.1590/S0102-311X2011000500005

9. Muller DK, Dias da Costa JS, Luz AMH, Olinto MTA. Cobertura do exame citopatológico do colo do útero na cidade de São Leopoldo, Rio Grande do Sul, Brasil. Cad Saude Publica. 2008;24(11):2511-20. https://doi.org/10.1590/S0102-311X2008001100006

10. Marmot M. Brazil: rapid progress and the challenge of inequality. Int J Equity Health. 2016;15(1):177. https://doi.org/10.1186/s12939-016-0465-y

11. Victora C. Socioeconomic inequalities in health: reflections on the academic production from Brazil. Int J Equity Health. 2016;15(1):164. https://doi.org/10.1186/s12939-016-0456-z

12. Macinko J, Harris MJ, Phil D. Brazil's Family Health Strategy: delivering community-based primary care in a universal health system. N Eng/ J Med. 2015;372(23):2177-81. https://doi.org/10.1056/NEJMp1501140.

13. Boing AF, Matos IB, Arruda MP, Oliveira MC, Njaine K. Prevalência de consultas médicas e fatores associados: um estudo de base populacional no sul do Brasil Rev Assoc Med Bras. 2010;56(1):41-6. https://doi.org/10.1590/S0104-42302010000100014

14. Bastos GAN, Harzheim E, Sousa AI. Prevalência e fatores associados à consulta médica entre adultos de uma comunidade de baixa renda do Sul do Brasil. Epidemiol Serv Saude. 2014;23(3):409-20. https://doi.org/10.5123/S1679-49742014000300004

15. Boccolini CS, Souza Júnior PRB. Inequities in healthcare utilization: results of the Brazilian National Health Survey, 2013. Int J Equity Health. 2016;15:150. https://doi.org/10.1186/s12939-016-0444-3

16. Bastos GAN, Del Ducan GF, Hallal PC, Santos IS. Utilização de serviços médicos no sistema público de saúde no Sul do Brasil. Rev Saude Publica. 2011;45(3):475-84. https://doi.org/10.1590/S0034-89102011005000024

17. Schmidt MI, Duncan BB, Azevedo e Silva G, Menezes AM, Monteiro CA, Barreto SM, et al. Chronic non-communicable diseases in Brazil: burden and current challenges. Lancet. 2011;377(9781):1949-61. https://doi.org/10.1016/S0140-6736(11)60135-9

18. Dias da Costa JS, Olinto MTA, Gigante DP, Menezes AMB, Macedo S, Daltoé T, et al. Utilização de serviços ambulatoriais de saúde em Pelotas, Rio Grande do Sul, Brasil: alguns fatores relacionados com as consultas médicas acima da média. Cad Saude Publica. 2008;24(2):353-63. https://doi.org/10.1590/S0102-311X2008000200014

19. Carvalho SC, Mota E, Dourado I, Aquino R, Teles C, Medina MG. Hospitalizations of children due to primary health care sensitive conditions in Pernambuco State, Northeast Brazil. Cad Saude Publica. 2015;31(4):744-54. https://doi.org/10.1590/0102-311X00069014

Contribuição dos Autores: Concepção e planejamento do estudo: JSDC, MTAO. Análise e interpretação dos dados: MB, JFL, TD, TM. Elaboração ou revisão do manuscrito: APK, BC, PBX. Aprovação da versão final: TD, PBX, TM. Responsabilidade pública pelo conteúdo do artigo: JSDC.

Conflito de Interesses: Os autores declaram não haver conflito de interesses. 